University of Nebraska - Lincoln

DigitalCommons@University of Nebraska - Lincoln

Faculty Publications, Department of Physics and Astronomy

Research Papers in Physics and Astronomy

July 2003

\title{
Static and dynamic dipole polarizability of the helium atom using wave functions involving logarithmic terms
}

Mauro Masili

Centro Universitário Central Paulista, UNICEP, São Paulo, Brazil

Anthony F. Starace

University of Nebraska-Lincoln, astarace1@unl.edu

Follow this and additional works at: https://digitalcommons.unl.edu/physicsfacpub

Part of the Physics Commons

Masili, Mauro and Starace, Anthony F., "Static and dynamic dipole polarizability of the helium atom using wave functions involving logarithmic terms" (2003). Faculty Publications, Department of Physics and Astronomy. 47.

https://digitalcommons.unl.edu/physicsfacpub/47

This Article is brought to you for free and open access by the Research Papers in Physics and Astronomy at DigitalCommons@University of Nebraska - Lincoln. It has been accepted for inclusion in Faculty Publications, Department of Physics and Astronomy by an authorized administrator of DigitalCommons@University of Nebraska Lincoln. 


\title{
Static and dynamic dipole polarizability of the helium atom using wave functions involving logarithmic terms
}

\author{
Mauro Masili \\ Centro Universitário Central Paulista, UNICEP, Rua Miguel Petroni 5111, 13 563-470 São Carlos, São Paulo, Brazil \\ Anthony F. Starace \\ Department of Physics and Astronomy, The University of Nebraska, 116 Brace Laboratory, Lincoln, Nebraska 68588-0111, USA
}

(Received 10 June 2002; revised manuscript received 15 May 2003; published 23 July 2003)

\begin{abstract}
We present a calculation of the static and dynamic dipole polarizability of the helium atom using a variationally stable treatment that incorporates the coupled-channel hyperspherical representation of the wave functions. Inclusion of logarithmic terms in intermediate functions as well as the effect of an optimization procedure for the variational parameter are analyzed. When available, our coupled-channel results are compared with other values in the literature.
\end{abstract}

DOI: 10.1103/PhysRevA.68.012508

PACS number(s): 32.10.Dk, 32.80.-t

\section{INTRODUCTION}

It was demonstrated long ago that the wave function for the helium atom at the triple-collision point should formally be described as a power series in $R$ and $\ln R$, where $R$ is the hyperspherical radius [1,2]. However, this description has only been applied in calculations of energy levels [3-7]. The main effect of this expansion is to speed up the convergence of the calculated energy, reducing the large number of basis functions needed in usual variational calculations. More recent works [8-14] have demonstrated that alternative kinds of expansions, not involving logarithmic terms, can achieve benchmark energy levels with reasonable basis sizes. These methods involve double $[9,11]$ and triple [12] basis sets, noninteger $[8]$ or complex $[13,14]$ powers of the expansion variables, or special kinds of configuration interaction expansions [10]. In a recent work, Popov and Ancarani [15] showed, in a rigorous mathematical study of the bound states of the helium atom, how the logarithmic terms, as suggested by Bartlett [1], are linked to the electron-electron interaction in the region of small radii. Nevertheless, they discussed only briefly possible numerical methods for getting approximate energy levels.

The dipole polarizability of helium is another fundamental property of this prototypical two-electron system whose accurate calculation has generated much interest (see, e.g., Refs. [16-27]). A comprehensive review on electric dipole polarizabilities for atoms has been given by Bonin and Kadar-Kallen [28]. As pointed out in that review, polarizabilities are important in a number of areas in physics and chemistry, such as interactions between matter and electromagnetic fields, collision phenomena, and others. Many physical properties are related to the polarizability, as for instance the dielectric constant and refractive index. Owing to such a broad interest in determining polarizabilities, many theoretical methods have been employed. However, many fewer highly accurate methods exist for polarizabilities than for energies. An interesting fact, recently shown by Pachucki and Sapirstein [27], is that the mass polarization, relativistic, and QED corrections to the nonrelativistic static polarizability cancel almost completely, giving a contribution to the $\mathrm{He}$ static polarizability of under $2 \mathrm{ppm}$. This accidental cancellation highlights the importance of highly accurate calculations of the polarizability in the nonrelativistic limit in order to test the effect of these higher-order corrections. Although many different methods and techniques exist, only a few of them are able to give the requisite precision. Significantly, none of the many prior calculations include logarithmic terms in their formulation.

We present, in addition to energy levels, a calculation of the static and dynamic dipole polarizability of the helium atom using a variationally stable, coupled-channel hyperspherical approach [20,29-32], in which the initial- and intermediate-state wave functions are represented by Fock expansions [1,2,33-36]. Our results include logarithmic terms in the wave-function expansions in order to calculate an observable other than the energy. In the calculation of energy levels, those terms have served only to speed up the convergence of the result (see, e.g., Refs. [4,5]). On the contrary, in our calculation of the dipole polarizability, the logarithmic terms play a crucial role in obtaining accurate values when using nonoptimized parameters in the intermediatestate functions. When an optimization procedure is included, the logarithmic terms become less important, at the expense of more CPU time. Comparing the results we obtain both with and without the logarithmic terms for the static polarizability, we are able to demonstrate their important role for the hyperspherical basis we employ. The present approach, in which we treat up to 12 coupled ${ }^{1} S^{e}$ channels and up to 15 coupled ${ }^{1} P^{o}$ channels, is able to furnish five digits of accuracy for polarizabilities. While this level of accuracy is unable to provide benchmark results for the He static polarizability (since the best results of others give seven or more digits of accuracy), our method is nevertheless more than sufficient to provide competitive results for the dynamical polarizabilities as well as for our next main goal, the calculation of multiphoton cross sections with an accuracy exceeding that of current experimental capabilities.

This paper is structured as follows. In Sec. II we summarize the theoretical aspects of the present approach, giving the fundamental equations. Section III gives some details regarding the numerical and calculational features of our ap- 
proach. In Sec. IV we present the results for the static and frequency-dependent dipole polarizabilities as well as for the energies; we provide also an analysis of the convergence of our results. In addition, comparisons with other results in literature are given. Finally, Sec. V presents our conclusions and some perspectives.

\section{THE VARIATIONALLY STABLE, COUPLED-CHANNEL HYPERSPHERICAL APPROACH}

For two-electron systems, such as helium and its isoelectronic series, the hyperspherical coordinate representation is very suitable for describing the wave functions (see Ref. [37] for a review on this subject). The set of coordinates employed is $\left\{R, \alpha, \theta_{1}, \phi_{1}, \theta_{2}, \phi_{2}\right\}$, where $R=\left(r_{1}^{2}+r_{2}^{2}\right)^{1 / 2}, \alpha$ $=\tan ^{-1}\left(r_{1} / r_{2}\right)$, and $\theta_{i}, \phi_{i}$ are the usual angular coordinates.

The matrix element for a two-photon transition between an initial state $|i\rangle$ and a final state $|f\rangle$ is written as

$$
T_{i \rightarrow f}^{(2)}(\omega)=\left\langle f\left|D \frac{1}{E_{i}+\omega-\hat{H}} D\right| i\right\rangle,
$$

where $D=\boldsymbol{\epsilon} \cdot\left(\mathbf{r}_{1}+\mathbf{r}_{2}\right)$ is the length form of the electric dipole operator, $E_{i}$ is the energy of the initial state, $\boldsymbol{\epsilon}$ is the light polarization vector, and $\omega$ is the photon energy. The variationally stable form of Eq. (1), according to Refs. $[20,29-31]$, is

$$
T_{i \rightarrow f}^{(2)}(\omega)=\langle f|D| \lambda\rangle+\left\langle\lambda^{\prime}|D| i\right\rangle-\left\langle\lambda^{\prime}\left|E_{i}+\omega-\hat{H}\right| \lambda\right\rangle,
$$

where $|\lambda\rangle$ and $\left\langle\lambda^{\prime}\right|$ represent unknown functions related to $|i\rangle$ and $\langle f|$, respectively, by a one-photon transition. The two-photon transition rate in Eq. (2) is variationally stable in the sense that it depends only quadratically on errors in the determination of $|\lambda\rangle$ and $\left\langle\lambda^{\prime}\right|$ [30]. In Eq. (2), the Hamiltonian in hyperspherical coordinates is given by (atomic units are used throughout this paper)

$$
\hat{H}=-\frac{1}{2}\left(\frac{\partial^{2}}{\partial R^{2}}+\frac{\hat{U}(R, \Omega)+1 / 4}{R^{2}}\right),
$$

and the operator $\hat{U}(R, \Omega)$ is the angular part of the Hamiltonian, with a parametric dependence on $R$,

$$
\begin{aligned}
\hat{U}(R, \Omega)= & \frac{\partial^{2}}{\partial \alpha^{2}}-\frac{\hat{L}_{1}^{2}}{\sin ^{2} \alpha}-\frac{\hat{L}_{2}^{2}}{\cos ^{2} \alpha}+\frac{2 Z R}{\sin \alpha}+\frac{2 Z R}{\cos \alpha} \\
& -\frac{2 R}{\sqrt{1-\sin (2 \alpha) \cos \theta_{12}}},
\end{aligned}
$$

where $\hat{L}_{1}^{2}$ and $\hat{L}_{2}^{2}$ are the usual angular-momentum operators of the individual electrons and $\theta_{12}$ is the angle between their position vectors: $\cos \theta_{12}=\hat{\mathbf{r}}_{1} \cdot \hat{\mathbf{r}}_{2}$. An eigenvalue equation for the operator $\hat{U}(R, \Omega)$, i.e.,

$$
\hat{U}(R, \Omega) \Phi_{\mu}(R ; \Omega)=U_{\mu}(R) \Phi_{\mu}(R ; \Omega),
$$

furnishes a set of channel functions $\Phi_{\mu}(R ; \Omega)$ and a set of corresponding potential curves $U_{\mu}(R)$, where the index $\mu$ is a collective label for all relevant quantum numbers. At $R$ $=0$, Eq. (5) is exactly solvable and the solutions can be written in terms of Jacobi polynomials with the corresponding eigenvalues given by

$$
U_{\mu}(0)=-\left(l_{1}+l_{2}+2 \tilde{n}+2\right)^{2},
$$

where $l_{1}$ and $l_{2}$ are the individual angular-momentum quantum numbers of the electrons and $\tilde{n}$ is the degree of the Jacobi polynomial of a particular solution. It represents the number of nodes in $\alpha$ (at $R=0$ ) of a given channel.

The initial- and final-state wave functions as well as the functions $\lambda$ and $\lambda^{\prime}$ are expanded in adiabatic hyperspherical channel functions $[20,38,31]$; they all have similar forms:

$$
\begin{gathered}
\Psi(R, \Omega)=\left(R^{5 / 2} \sin \alpha \cos \alpha\right)^{-1} \sum_{\mu} F_{\mu}(R) \Phi_{\mu}(R ; \Omega), \\
\lambda(R, \Omega)=\left(R^{5 / 2} \sin \alpha \cos \alpha\right)^{-1} \sum_{\nu} \lambda_{\nu}(R) \Phi_{\nu}(R ; \Omega), \\
\lambda^{\prime}(R, \Omega)=\left(R^{5 / 2} \sin \alpha \cos \alpha\right)^{-1} \sum_{\mu} \lambda_{\mu}^{\prime}(R) \Phi_{\mu}(R ; \Omega),
\end{gathered}
$$

where the expansion coefficients $F_{\mu}(R)$ satisfy the coupled radial equations

$$
\begin{aligned}
\left(\frac{d^{2}}{d R^{2}}\right. & \left.+\frac{U_{\mu}(R)+1 / 4}{R^{2}}+2 E\right) F_{\mu}(R)+\sum_{\nu}\left[2 P_{\mu \nu}(R) \frac{d}{d R}\right. \\
& \left.+Q_{\mu \nu}(R)\right] F_{\nu}(R)=0
\end{aligned}
$$

and $\lambda_{\nu}(R)$ and $\lambda_{\mu}^{\prime}(R)$ are determined by the variational procedure described below. The coupling terms in Eq. (10) are known as nonadiabatic couplings and are defined by

$$
P_{\mu \nu}(R)=\left\langle\Phi_{\mu}\left|\frac{d}{d R}\right| \Phi_{\nu}\right\rangle
$$

and

$$
Q_{\mu \nu}(R)=\left\langle\Phi_{\mu}\left|\frac{d^{2}}{d R^{2}}\right| \Phi_{\nu}\right\rangle,
$$

where the brackets mean integration over the angular variables $\Omega$. In order to evaluate the radial integrals of Eq. (2), we expand the unknown radial functions as

$$
\lambda_{\nu}(R)=\sum_{i=1}^{B_{\max }} \sum_{j=1}^{M_{i}} a_{i j}^{\nu} \phi_{i j}^{\nu}(R)
$$




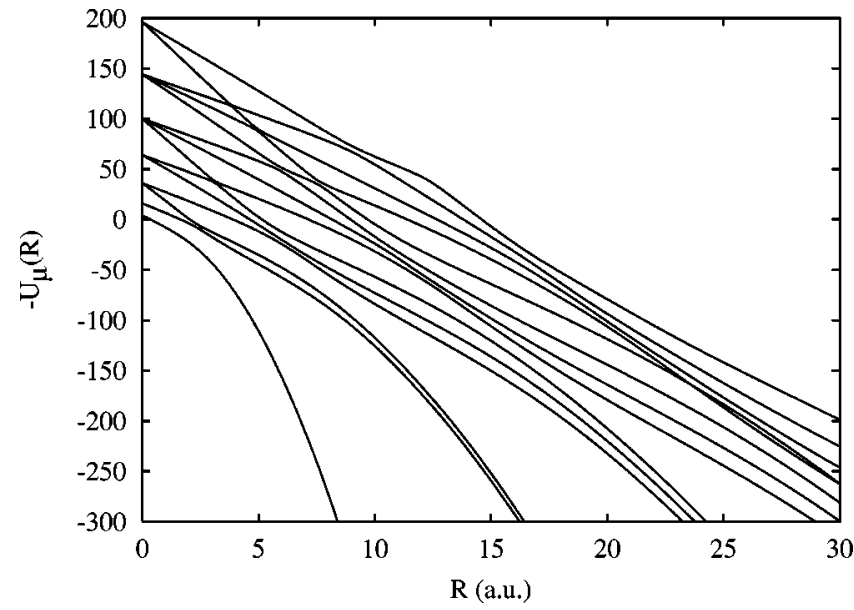

FIG. 1. Set of potential curves $-U_{\mu}(R)$ for ${ }^{1} S^{e}$ states of the helium atom.

$$
\lambda_{\mu}^{\prime}(R)=\sum_{i^{\prime}=1}^{B_{\max }} \sum_{j^{\prime}=1}^{M_{i^{\prime}}} b_{i^{\prime} j^{\prime}}^{\mu} \theta_{i^{\prime} j^{\prime}}^{\mu}(R),
$$

where $\phi_{i j}^{\nu}(R)$ and $\theta_{i^{\prime} j^{\prime}}^{\mu}(R)$ are chosen to be modified Slater orbitals that include powers of $\ln R$, i.e.,

$$
\phi_{i j}^{\nu}(R)=N_{i, j+2(i-1)}^{\nu} R^{S_{i j}^{\nu}(\ln R)^{i-1}} e^{-\beta_{i}^{\nu} R}
$$

and

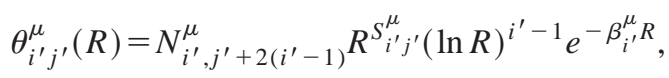

where $N_{i, j}^{\mu}$ is a normalization constant for the $i, j$ basis function of each channel $\mu$ and the exponents of $R$ are given by $S_{i j}^{\mu}=\sqrt{-U_{\mu}(0)}+1 / 2+j+2(i-1)$ (cf. Ref. [35]). The constants $\beta_{i}^{\mu}$ are free parameters whose determination is described in Sec. III.

The frequency-dependent polarizability is calculated using the second-order transition matrix element as follows:

$$
\alpha(\omega)=-\left[T_{i \rightarrow i}^{(2)}(+\omega)+T_{i \rightarrow i}^{(2)}(-\omega)\right],
$$

where the final state $|f\rangle$ is replaced by the initial state $|i\rangle$ in Eq. (2). In the static limit, i.e., the photon frequency $\omega$ $\rightarrow 0$, the expression for the polarizability reduces to a simpler form: $\alpha(0)=-2 T_{i \rightarrow i}^{(2)}(0)$.

\section{CALCULATIONAL ASPECTS}

In this section, we discuss the numerical aspects of our calculations. It should be stressed here that these are numerically intensive calculations, particularly because our codes use REAL*16 (quadruple) precision in order to minimize numerical error propagation and to deal accurately with a mix of both small and large numbers.

In Eqs. (7)-(12), the channel indices $\mu$ and $\nu$ run from 1 to a maximum number $N_{c}$ of coupled channels that are considered in the adiabatic expansions. The positive constants $\beta_{i}^{\mu}$, introduced through the Slater basis functions in Eqs. (15) and (16), can be heuristically chosen: the starting trial

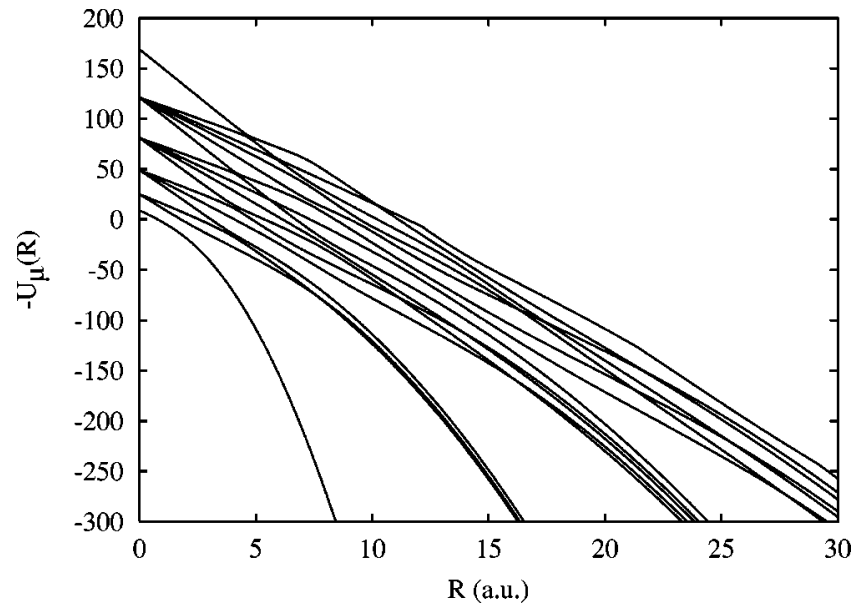

FIG. 2. Same as Fig. 1 for ${ }^{1} P^{o}$ states.

values for the $\beta_{i}^{\mu}$ are chosen to be close to the value $\sqrt{-2 E_{0}}$, where $-E_{0}=0.903724$ a.u. is the electron binding energy for the helium atom. Then, a spread of values around the initial trial values are investigated and their convergence properties and stability are examined with the aim of minimizing error propagation. In this work, we have also developed an optimization procedure for searching for the best set of $\beta_{i}^{\mu}$ parameters. This procedure is based on the minimization of the transition matrix element [Eq. (2)] with respect to $\beta_{i}^{\mu}$. Note that for photon frequencies above the ionization threshold, $\beta_{i}^{\mu}$ should be chosen to be complex in order to correctly describe the oscillatory character of the intermediate-state continuum wave functions.

The hyperspherical method focuses on the determination of potential curves and the corresponding channel functions [see Eq. (5)]. Due to the nature of the angular operator [Eq. (4)], the solution of Eq. (5) is the most difficult step of the method. Nevertheless, the angular operator $\hat{U}(R, \Omega)$ is not dependent on the system's energies, which means that the set of potential curves and nonadiabatic couplings are calculated a single time for the system under consideration. Due to the symmetry breaking caused by the electron-electron repulsion, the angular operator is not separable and Eq. (5) becomes an angular coupled channel equation [38]. In solving this eigenvalue equation, we have used an expansion in the individual angular momenta of the electrons [35]. The coupled-channel expansions have been truncated at the maximum value $l_{1}^{\max }=l_{2}^{\max }=9$ for the ${ }^{1} S^{e}$ and ${ }^{1} P^{o}$ channels with the exception of the lowest potential curves of each symmetry. These potential curves are the most important ones since they support the bound states. For the first ${ }^{1} S^{e}$ potential curve, 40 components of angular momentum have been included, i.e., $l_{1}^{\max }=l_{2}^{\max }=39$ and for the first ${ }^{1} P^{o}$ potential curve, 60 components have been used, i.e., $l_{1}^{\max }=l_{2}^{\max }=30$. Bound and scattered states are obtained using proper boundary conditions in solving the radial equations. In the calculation of static and dynamic polarizabilities (taking the initial state as the ground state), potential curves for both ${ }^{1} S^{e}$ and ${ }^{1} P^{o}$ states are required. 
TABLE I. Ground-state energy convergence as a function of the number $N_{c}$ of coupled radial equations. The error is relative to the variational value of Frankowski and Pekeris [4]. The first row, with $N_{c}=1$, corresponds to the calculation in which all couplings are neglected, giving a lower-energy bound; the second row, also with $N_{c}=1$, corresponds to the one in which only the diagonal coupling matrix element is taken into account, giving an upper-energy bound. Horizontal lines delimit groups of channels (see text).

\begin{tabular}{cccc}
\hline \hline$N_{c}$ & $\left(l_{1}, l_{2}, \tilde{n} ; n\right)$ & $E_{i}($ a.u. $)$ & $\left(E_{\mathrm{var}}-E_{i}\right) / E_{\mathrm{var}}(\mathrm{ppm})$ \\
\hline 1 & $(0,0,0 ; 1)$ & -2.930032616 & -9060.171 \\
1 & $(0,0,0 ; 1)$ & -2.895554014 & 2813.753 \\
2 & $(1,1,0 ; 2)$ & -2.898646614 & 1748.707 \\
\hline 3 & $(0,0,2 ; 2)$ & -2.903611486 & 38.878 \\
4 & $(2,2,0 ; 3)$ & -2.903632473 & 31.650 \\
5 & $(1,1,2 ; 3)$ & -2.903636415 & 30.293 \\
6 & $(3,3,0 ; 3)$ & -2.903658492 & 22.690 \\
\hline 7 & $(0,0,4 ; 4)$ & -2.903717088 & 2.510 \\
8 & $(2,2,2 ; 4)$ & -2.903717136 & 2.494 \\
9 & $(4,4,0 ; 4)$ & -2.903717238 & 2.459 \\
10 & $(1,1,4 ; 4)$ & -2.903717274 & 2.411 \\
11 & $(3,3,2 ; 5)$ & -2.903717376 & 2.197 \\
12 & $(5,5,0 ; 5)$ & -2.903717997 & 0.481 \\
\hline 13 & $(0,0,6 ; 5)$ & -2.903722980 & 0.465 \\
14 & $(2,2,4 ; 5)$ & -2.903723027 & \\
\hline Variational value $[4]$ & & -2.903724377 & \\
\hline \hline
\end{tabular}

\section{RESULTS}

The potential curves used in our calculations are shown in Figs. 1 and 2, where the $R=0$ degeneracy is evident and exact, according to Eq. (6). The degenerate curves suggest a natural grouping of channels, which will prove relevant for analyzing the convergence of our results as a function of the number of channels included in our calculations. Note the apparent crossings between contiguous curves, especially for the higher ones. In fact, those are avoided-crossing regions, where the corresponding channel functions suddenly ex-

TABLE II. Same as Table I, for the $1 s 2 p{ }^{1} P^{o}$ state, where the error is relative to the variational value of Schiff et al. [43]. Horizontal lines delimit groups of channels (see text).

\begin{tabular}{cccc}
\hline \hline$N_{c}$ & $\left(l_{1}, l_{2}, \tilde{n} ; n\right)$ & $E_{i}($ a.u. $)$ & $\left(E_{\mathrm{var}}-E_{i}\right) / E_{\mathrm{var}}(\mathrm{ppm})$ \\
\hline 1 & $(0,1,0 ; 1)$ & -2.145599305 & -10243.798 \\
1 & $(0,1,0 ; 1)$ & -2.121696638 & 1010.643 \\
\hline 2 & $(0,1,1 ; 2)$ & -2.123010231 & 392.145 \\
3 & $(1,2,0 ; 2)$ & -2.123219358 & 293.679 \\
\hline 4 & $(0,1,2 ; 2)$ & -2.123611967 & 108.821 \\
5 & $(1,2,1 ; 3)$ & -2.123621908 & 104.141 \\
6 & $(2,3,0 ; 3)$ & -2.123645237 & 93.156 \\
\hline 7 & $(0,1,3 ; 3)$ & -2.123734421 & 51.165 \\
8 & $(1,2,2 ; 3)$ & -2.123738246 & 49.363 \\
9 & $(2,3,1 ; 3)$ & -2.123750987 & 41.377 \\
10 & $(3,4,0 ; 4)$ & -2.123755209 & 24.639 \\
\hline 11 & $(0,1,4 ; 4)$ & -2.123790757 & 24.045 \\
12 & $(1,2,3 ; 4)$ & -2.123792018 & 22.671 \\
13 & $(2,3,2 ; 4)$ & -2.123794936 & 21.455 \\
14 & $(3,4,1 ; 4)$ & -2.123797519 & 20.975 \\
15 & $(4,5,0 ; 4)$ & -2.123798539 & 15.053 \\
\hline 16 & $(0,1,5 ; 4)$ & -2.123811116 & \\
\hline \hline
\end{tabular}


TABLE III. Static polarizability of the helium atom using five- $\beta$ Slater basis functions without and with logarithmic terms (for $N_{c}$ $=N_{c}^{\prime}$ ), where $N_{c}$ is the number of coupled channels. The difference between the two rows with $N_{c}=1$ is explained in the caption of Table I. The results for $\alpha(0)$ in columns 4 and 5 do not involve optimized values of the $\beta_{i}^{\mu}$ parameters; those in columns 6 and 7 do.

\begin{tabular}{|c|c|c|c|c|c|c|}
\hline \multirow[b]{2}{*}{$N_{c}$} & \multirow[b]{2}{*}{$\begin{array}{c}S \text { state } \\
\left(l_{1}, l_{2}, \tilde{n} ; n\right)\end{array}$} & \multirow[b]{2}{*}{$\begin{array}{c}P \text { state } \\
\left(l_{1}, l_{2}, \tilde{n} ; n\right)\end{array}$} & \multicolumn{4}{|c|}{$\alpha(0)$} \\
\hline & & & $\begin{array}{l}\text { Neglecting } \\
\log \text { terms }{ }^{\text {a }}\end{array}$ & $\begin{array}{l}\text { Including } \\
\log \text { terms }{ }^{\text {a }}\end{array}$ & $\begin{array}{c}\text { Optimized } \\
\text { without } \log \text { terms }\end{array}$ & $\begin{array}{c}\text { Optimized } \\
\text { with } \log \text { terms }\end{array}$ \\
\hline 1 & $(0,0,0 ; 1)$ & $(0,1,0 ; 1)$ & 1.386392 & 1.386392 & & \\
\hline 1 & $(0,0,0 ; 1)$ & $(0,1,0 ; 1)$ & 1.394070 & 1.394070 & 1.39406870 & 1.39406870 \\
\hline 2 & $(1,1,0 ; 2)$ & $(0,1,1 ; 2)$ & 1.381100 & 1.395580 & 1.39557602 & 1.39557603 \\
\hline 3 & $(0,0,2 ; 2)$ & $(1,2,0 ; 2)$ & 1.356458 & 1.380733 & 1.38073090 & 1.38073132 \\
\hline 4 & $(2,2,0 ; 3)$ & $(0,1,2 ; 2)$ & 1.387758 & 1.383117 & 1.38311607 & 1.38311644 \\
\hline 5 & $(1,1,2 ; 3)$ & $(1,2,1 ; 3)$ & 1.387457 & 1.382986 & 1.38297307 & 1.38297312 \\
\hline 6 & $(3,3,0 ; 3)$ & $(2,3,0 ; 3)$ & 1.387635 & 1.383227 & 1.38322532 & 1.38322555 \\
\hline 7 & $(0,0,4 ; 4)$ & $(0,1,3 ; 3)$ & 1.392171 & 1.383045 & 1.38306640 & 1.38306730 \\
\hline 8 & $(2,2,2 ; 4)$ & $(1,2,2 ; 3)$ & 1.393330 & 1.383086 & 1.38306882 & 1.38306973 \\
\hline 9 & $(4,4,0 ; 4)$ & $(2,3,1 ; 3)$ & 1.392195 & 1.383067 & 1.38306974 & 1.38307043 \\
\hline 10 & $(1,1,4 ; 4)$ & $(3,4,0 ; 4)$ & 1.392073 & 1.383094 & 1.38307442 & 1.38307430 \\
\hline 11 & $(3,3,2 ; 5)$ & $(0,1,4 ; 4)$ & 1.397085 & 1.383163 & 1.38315243 & 1.38315273 \\
\hline 12 & $(5,5,0 ; 5)$ & $(1,2,3 ; 4)$ & 1.391545 & 1.383193 & 1.38316264 & 1.38316294 \\
\hline
\end{tabular}

${ }^{\mathrm{a}}$ Five nonoptimized $\beta_{i}^{\mu}$ parameters.

${ }^{\mathrm{b}}$ One $\beta_{i}^{\mu}$ parameter. Results from Table IV.

${ }^{\mathrm{c}}$ Two $\beta_{i}^{\mu}$ parameters.

change their behaviors, resulting in sharp peaks in the nonadiabatic couplings due to the first and second derivatives present in their definitions, as seen in Eqs. (11) and (12).

For reasons of consistency, the ground-state energy $E_{i}$ used in Eq. (1) is the calculated hyperspherical value for the number of channels included in our calculations, as shown in Table I. In this table, as the number of coupled channels increases, the corresponding calculated energy approaches the variational value of Ref. [4]. The first row with $N_{c}=1$ corresponds to the calculation in which all couplings are neglected, giving a lower bound for the energy $[39,40]$. The second row with $N_{c}=1$ corresponds to the calculation in which only the diagonal coupling is taken into account. This value, and all subsequent ones, are upper-energy bounds $[39,40]$. The second column lists the $R=0$ angular quantum numbers [see Eq. (6)] as well as the asymptotic hydrogenic threshold $n$; this set of numbers labels each potential curve (and the corresponding angular channel). For second-order processes such as the dipole polarizability of the ground state, the intermediate states possess symmetry ${ }^{1} P^{o}$. For this reason, Table II is included in order to analyze the convergence of the $1 s 2 p$ energy. One sees in Table I that as additional adiabatic hyperspherical channels are included in our calculations, the deviations from the variational value of Ref. [4] decrease monotonically. Large decreases occur when the hyperspherical channels that converge to $n=2\left(N_{c}=2,3\right)$, $n=3\left(N_{c}=4,5,6\right)$, and the first channel converging to $n$ $=4\left(N_{c}=7\right)$ are included. The largest decreases occur when the last channel included has an angular function containing $s$ waves (i.e., electron orbital angular momenta $l_{1}=l_{2}=0$ ) at $R=0$; this occurs for $N_{c}=3,7$, and 13. A similar pattern can be found in Table II for the $1 s 2 p$ state. In both Tables I and
II, horizontal lines delimit groups of channels. Inside each group the convergence is steady but slow. One could also try grouping of channels using the asymptotic threshold $n$ as a parameter. We believe that grouping of channels using their $R=0$ characters is the most appropriate method since the corresponding potential wells [see Eq. (3)] are in the smalland mid- $R$ regions and, consequently, in this region the channel functions are better characterized by their $R=0$ behaviors than by their $R \rightarrow \infty$ behaviors. As noted from Tables I and II, a new group of channels starts when an angular channel with an $l_{1}=0$ component is included. Note how every time a new channel having $l_{1}=0$ is included, the error drops dramatically. This behavior (and its associated channel grouping) will prove useful for analyzing the convergence of the static polarizability, for which both ${ }^{1} S^{e}$ and ${ }^{1} P^{o}$ symmetries are involved.

\section{A. Static polarizability}

In our calculation of the static polarizability without the optimization of the exponential parameters $\beta_{i}^{\mu}$, our expansions of the radial functions in Eqs. (15) and (16) include factors such as $R^{S_{i 0}^{\mu}+j}(\ln R)^{i-1}$, where $1 \leqslant i \leqslant B_{\max }$ and $1 \leqslant j \leqslant M_{i}$ so that $B_{\max }-1$ is the highest power of $\ln R$ and $M_{i}+S_{i 0}^{\mu}$ is the highest power of $R$ for a given power ( $i$ $-1)$ of $\ln R$. We have used in the expansions of Eqs. (13) and (14), $B_{\max }=5$ with $\beta_{1}^{\mu}=0.3, \beta_{2}^{\mu}=0.5, \beta_{3}^{\mu}=0.8, \beta_{4}^{\mu}$ $=1.2$, and $\beta_{5}^{\mu}=1.7$. For each value of $\beta_{i}^{\mu}, M_{i}=M=12$, although $M=8$ or even $M=6$ suffices to obtain reasonable precision. Although for $\mathrm{H}^{-}$we have found that $B_{\max }=1$ suffices [32], which implies the absence of logarithmic terms in 
TABLE IV. Convergence for the calculated static dipole polarizability of the helium atom as a function of the number of coupled channels for the initial ground state $\left(N_{c}\right)$ and for the intermediate states $\left(N_{c}^{\prime}\right)$. Calculations employ one optimized $\beta_{i}^{\mu}$ parameter. Horizontal and vertical lines delimit groups of channels (see text).

\begin{tabular}{|c|c|c|c|c|c|c|c|c|c|c|c|c|c|c|}
\hline \multicolumn{15}{|c|}{$N_{c}$} \\
\hline$N_{c}^{\prime}$ & 1 & 2 & 3 & 4 & 5 & 6 & 7 & 8 & 9 & 10 & 11 & 12 & 13 & 14 \\
\hline 1 & 1.394069 & 1.353977 & 1.324336 & 1.324337 & 1.324098 & 1.324162 & 1.323510 & 1.323511 & 1.323510 & 1.323504 & 1.323510 & 1.323519 & 1.323460 & 1.323460 \\
\hline 2 & 1.427833 & 1.395576 & 1.380032 & 1.379974 & 1.379764 & 1.379803 & 1.379160 & 1.379160 & 1.379160 & 1.379154 & 1.379159 & 1.379169 & 1.379110 & 1.379110 \\
\hline 3 & 1.428627 & 1.395777 & 1.380731 & 1.380723 & 1.380515 & 1.380555 & 1.379910 & 1.379911 & 1.379910 & 1.379905 & 1.379910 & 1.379919 & 1.379861 & 1.379861 \\
\hline 4 & 1.431625 & 1.398484 & 1.383111 & 1.383116 & 1.382930 & 1.382980 & 1.382345 & 1.382346 & 1.382345 & 1.382340 & 1.382345 & 1.382354 & 1.382297 & 1.382297 \\
\hline 5 & 1.431669 & 1.398502 & 1.383140 & 1.383149 & 1.382973 & 1.383022 & 1.382387 & 1.382388 & 1.382387 & 1.382382 & 1.382387 & 1.382397 & 1.382341 & 1.382341 \\
\hline 6 & 1.431888 & 1.398665 & 1.383275 & 1.383302 & 1.383126 & 1.383225 & 1.382588 & 1.382589 & 1.382588 & 1.382583 & 1.382588 & \begin{tabular}{|l|}
.382598 \\
\end{tabular} & 1.382543 & 1.382543 \\
\hline 7 & 1.432474 & 1.399150 & 1.383652 & 1.383672 & 1.383483 & 1.383645 & 1.383066 & 1.383067 & 1.383066 & 1.383061 & 1.383066 & 1.383076 & 1.383021 & 1.383021 \\
\hline 8 & 1.432479 & 1.399152 & 1.383655 & 1.383675 & 1.383484 & 1.383647 & 1.383068 & 1.383069 & 1.383068 & 1.383063 & 1.383068 & 1.383077 & 1.383023 & 1.383023 \\
\hline 9 & 1.432480 & 1.399153 & 1.383656 & 1.383676 & 1.383485 & 1.383648 & 1.383070 & 1.383070 & 1.383070 & 1.383064 & 1.383070 & 1.383079 & 1.383024 & 1.383024 \\
\hline 10 & 1.432484 & 1.399158 & 1.383660 & 1.383680 & 1.383490 & 1.383658 & 1.383079 & 1.383080 & 1.383080 & 1.383074 & 1.383080 & 1.383089 & 1.383034 & 1.383034 \\
\hline 11 & 1.432585 & 1.399247 & 1.383736 & 1.383756 & 1.383565 & 1.383737 & 1.383152 & 1.383153 & 1.383152 & 1.383147 & 1.383152 & 1.383162 & 1.383107 & 1.383107 \\
\hline 12 & 1.432586 & 1.399247 & 1.383736 & 1.383756 & 1.383566 & 1.383738 & 1.383153 & 1.383153 & 1.383153 & 1.383148 & 1.383153 & 1.383163 & 1.383108 & 1.383108 \\
\hline 13 & 1.432588 & 1.399249 & 1.383738 & 1.383757 & 1.383567 & 1.383739 & 1.383154 & 1.383155 & 1.383154 & 1.383149 & 1.383155 & 1.383164 & 1.383109 & 1.383109 \\
\hline 14 & 1.432590 & 1.399250 & 1.383739 & 1.383759 & 1.383568 & 1.383740 & 1.383155 & 1.383156 & 1.383155 & 1.383150 & 1.383156 & 1.383165 & 1.383110 & 1.383110 \\
\hline 15 & 1.432601 & 1.399259 & 1.383747 & 1.383767 & 1.383576 & 1.383747 & 1.383162 & 1.383163 & 1.383162 & 1.383157 & 1.383164 & \begin{tabular}{|l|l|}
1.383174 \\
\end{tabular} & 1.383119 & 1.383119 \\
\hline 16 & 1.432621 & 1.399278 & 1.383763 & 1.383783 & 1.383593 & 1.383764 & 1.383179 & 1.383179 & 1.383179 & 1.383174 & 1.383196 & 1.383208 & 1.383157 & 1.383157 \\
\hline
\end{tabular}


TABLE V. Comparison of the present variationally stable results for the ground-state static dipole polarizability of the He atom with theoretical results of other authors and available experimental values. The result in the first line was calculated using $N_{c}=N_{c}^{\prime}=12$, whereas the two subsequent lines show results using $N_{c}=12$ and $N_{c}^{\prime}=15$.

\begin{tabular}{|c|c|}
\hline Reference & $\alpha(0)$ \\
\hline \multicolumn{2}{|c|}{ Theoretical results } \\
\hline This work (coupled-channel result including $\log$ terms ${ }^{\mathrm{a}}$ ) & 1.383193 \\
\hline This work (optimized coupled-channel result without log terms ${ }^{b}$ ) & 1.38317359 \\
\hline This work (optimized coupled-channel result including $\log$ terms ${ }^{\mathrm{c}}$ ) & 1.38317394 \\
\hline K. Pachucki and J. Sapirstein (2000) (Ref. [27]) & 1.383192174 \\
\hline Z.-C. Yan et al. (1996) (Ref. [25]) & 1.3831925 \\
\hline M. J. Jamieson, G. W. F. Drake, and A. Dalgarno (1995) (Ref. [24]) & 1.383192 \\
\hline A. K. Bhatia and R. J. Drachman (1994) (Ref. [23]) & 1.383192179 \\
\hline D. M. Bishop and J. Pipin (1993) (Ref. [22]) & 1.383192 \\
\hline D. M. Bishop and B. Lam (1988) (Ref. [19]) & 1.383192 \\
\hline R. M. Glover and F. Weinhold's lower bound (1976) (Ref. [17]) & 1.38259 \\
\hline R. M. Glover and F. Weinhold's upper bound (1976) (Ref. [17]) & 1.38411 \\
\hline S. J. A. van Gisbergen et al. (1998) (Ref. [26]) & 1.3824 \\
\hline H. P. Saha and C. D. Caldwell (1991) (Ref. [21]) & 1.374 \\
\hline B. Gao et al. (1990) (Ref. [20]) & 1.3559 \\
\hline E.-A. Reinsch (1985) MC-SCF result (Ref. [18]) & 1.383 \\
\hline K. T. Chung (1968) (Ref. [16]) & 1.3841 \\
\hline \multicolumn{2}{|c|}{ Experimental results } \\
\hline C. R. Mansfield and E. R. Peck (1969) (Ref. [44]) & 1.3861 \\
\hline P. W. Langhoff and M. Karplus (1969) (Ref. [45]) & 1.3838 \\
\hline D. Gugan and G. W. Michel (1980) (Ref. [46]) & $1.38377(7)$ \\
\hline D. Gugan (1991) (Ref. [47]) & $1.38379(4)$ \\
\hline K. Grohmann and H. Luther (1992) (Ref. [48]) & $1.383746(7)$ \\
\hline
\end{tabular}

${ }^{a}$ Five nonoptimized $\beta_{i}^{\mu}$ parameters, $N_{c}=N_{c}^{\prime}=12$ channels (cf. Table III).

${ }^{\mathrm{b}}$ One optimized $\beta_{i}^{\mu}$ parameter, $N_{c}=12, N_{c}^{\prime}=15$ channels (cf. Table IV).

${ }^{\mathrm{c}}$ Two optimized $\beta_{i}^{\mu}$ parameters, $N_{c}=12, N_{c}^{\prime}=15$ channels.

the intermediate-state functions, for the helium atom we discovered that not only should $B_{\max }$ be greater than unity but also that it needs to be at least greater than or equal to three in order to obtain convergence to the correct value of the static polarizability. In the adiabatic approximation, where $N_{c}=1$, the three matrix elements in Eq. (2) are equal to each other (to ten digits of accuracy). For the nonadiabatic coupled-channel calculation, i.e., $N_{c} \geqslant 2$, they are typically converged to within five or six digits of accuracy. We regard the accuracies of these equalities as indicators of the level of accuracy of our results. Note also that for these calculations we have not altered the parameters as the number of channels is increased; doing so may be necessary for larger numbers of channels, as these correspond to higher-energy levels of $\mathrm{He}^{+}$.

Table III lists the calculated values of the static polarizability according to Eq. (17), with $\omega=0$, as the number of coupled channels increases. The quantum numbers that label the potential curves, listed in Tables I and II, are also included to help identify the groups of channels. This table lists the values obtained by neglecting the logarithmic terms in Eqs. (15) and (16) alongside the results obtained by including the logarithmic terms. Other than the inclusion or exclusion of the logarithmic terms, both calculations were otherwise the same. That is, they used the same parameters (such as the number of $\beta_{i}^{\mu}$ constants) and the same number of basis functions. Comparison of the two variational calculations clearly indicates the importance of the use of the logarithmic expansion. They are important not only for accelerating the convergence but also for obtaining the correct value. Note that in calculating the polarizability there are two sets of coupled channels: the ${ }^{1} S^{e}$ channels, which couple to the ground state, and the ${ }^{1} P^{o}$ channels which constitute the intermediate states. One sees from Table III that the convergence is not a monotonically decreasing function of the number $N_{c}$ of coupled channels. An interesting question is whether the convergence would become smoother if different numbers of channels were coupled in ground and intermediate states. For example, one might include all hyperspherical channels that converge to a particular level $n$ of the $\mathrm{He}^{+}$ion in both ground and intermediate states. Alternatively, one might group the various adiabatic hyperspherical channels in some other way, such as according to their character at $R$ $=0$. We have carried out this study coupling all available channels in ground and intermediate states. In Table IV, we study the convergence of the static polarizability as a function of $N_{c}$ and $N_{c}^{\prime}$, which are the numbers of coupled channels in both ground and intermediate states, respectively, where we have used for this purpose a single $\beta_{i}^{\mu}$, which 
TABLE VI. Selected values of our best calculated $\left(N_{c}=N_{c}^{\prime}=6\right)$ dynamic polarizability of helium compared with results from other authors and with experimental values.

\begin{tabular}{|c|c|c|c|c|c|c|}
\hline$\omega$ & This work & Ref. [16] & Ref. [17] & Ref. [18] & Ref. [19] & Expt. $^{\mathrm{a}}$ \\
\hline 0.050 & 1.387094 & 1.3868 & 1.38722 & & 1.387066 & \\
\hline 0.100 & 1.398857 & 1.3990 & 1.39898 & 1.398 & 1.398820 & 1.399 \\
\hline 0.150 & 1.418998 & 1.4192 & 1.41912 & & 1.418957 & \\
\hline 0.200 & 1.448388 & 1.4483 & 1.44851 & 1.448 & 1.448341 & 1.449 \\
\hline 0.250 & 1.488389 & 1.4887 & 1.48853 & & 1.488335 & \\
\hline 0.300 & 1.541045 & 1.5407 & 1.54119 & 1.540 & 1.540981 & 1.542 \\
\hline 0.350 & 1.609399 & 1.6095 & 1.60956 & & 1.609325 & \\
\hline 0.400 & 1.698070 & 1.6980 & 1.6983 & 1.696 & 1.697985 & 1.700 \\
\hline 0.450 & 1.814308 & 1.8147 & 1.8145 & & 1.814214 & \\
\hline 0.500 & 1.970129 & 1.9706 & 1.9705 & 1.966 & 1.970037 & 1.973 \\
\hline 0.550 & 2.187047 & 2.1872 & 2.1875 & 2.182 & 2.186990 & \\
\hline 0.600 & 2.508200 & 2.5091 & 2.5091 & 2.501 & 2.508292 & 2.502 \\
\hline 0.650 & 3.036655 & 3.0380 & 3.0391 & 3.022 & 3.037345 & \\
\hline 0.700 & 4.107153 & 4.1103 & 4.1184 & 4.079 & 4.111021 & 3.884 \\
\hline 0.750 & 7.967789 & 7.9684 & 8.1640 & 7.967 & 8.014127 & \\
\hline 0.770 & 17.070652 & 16.8668 & & & & \\
\hline 0.780 & 1765.866727 & 56.0969 & -1073.68 & & & \\
\hline 0.782 & -90.326704 & 116.4560 & & & & \\
\hline 0.784 & -42.857302 & -968.8395 & & & & \\
\hline 0.785 & -33.466465 & & -31.46 & & & \\
\hline 0.790 & -14.990171 & -30.7461 & -14.56 & & & \\
\hline 0.795 & -8.852173 & & -8.71 & & & \\
\hline 0.800 & -5.722310 & -10.3290 & -5.66 & & & \\
\hline 0.805 & -3.768979 & & -3.75 & & & \\
\hline 0.810 & -2.380470 & & -2.38 & & & \\
\hline 0.815 & -1.286429 & & -1.30 & & & \\
\hline 0.820 & -0.336927 & & -0.37 & & & \\
\hline 0.825 & 0.578338 & & 0.52 & & & \\
\hline 0.830 & 1.581768 & & 1.47 & & & \\
\hline 0.835 & 2.894517 & & 2.65 & & & \\
\hline 0.840 & 5.173656 & & 4.52 & & & \\
\hline 0.845 & 12.633684 & & 9.75 & & & \\
\hline
\end{tabular}

${ }^{\mathrm{a}}$ Reference [18].

means that the basis functions do not include logarithmic terms. The horizontal and vertical lines correspond to the horizontal lines of Tables I and II, i.e., they show the beginning of each group of channels, where each group begins with a channel having $l_{1}=0$ at $R=0$ [see Eq. (6)]. In our calculations for $\alpha(0)$, we have introduced an optimization procedure for the parameters $\beta_{i}^{\mu}$. The optimal set of $\beta_{i}^{\mu}$ parameters is found by minimizing the transition matrix element [Eq. (2)] with respect to each $\beta_{i}^{\mu}$. Table III lists the results using optimized $\beta_{i}^{\mu}$ in the intermediate functions. A side-by-side comparison shows that only a single optimized value for the $\beta_{i}^{\mu}$ parameter is sufficient to achieve results very close to the ones obtained with five nonoptimized $\beta_{i}^{\mu}$ parameters and logarithmic terms. On the other hand, additional CPU time is required for the optimization procedure. Anticipating that readers will wish to know what would happen if one combines the inclusion of logarithmic terms together with the optimization of the $\beta_{i}^{\mu}$, a sample for the first few coupled channels have been computed and displayed in the last column of Table III. One sees that the improvement is beyond the sixth or seventh decimal digit, with a high cost in terms of computer resources.

Some information about the convergence of the calculated $\alpha(0)$ value with the number of channels can be extracted from Table IV. For each column, the same convergence pattern of ${ }^{1} P^{o}$ states, shown in Table II, is found as $N_{c}^{\prime}$ increases. Within each group of channels the polarizability increases slowly and monotonically whereas larger increments occur when a new group is included. A different pattern is noted when looking at the rows, which show the convergence as the number $N_{c}$ of channels included in the groundstate wave function is increased. Within each group the convergence oscillates with increasing $N_{c}$. However, the important feature is that considering only the values obtained with a complete group of $N_{c}$ channels included, the results are monotonically decreasing. These results are represented 

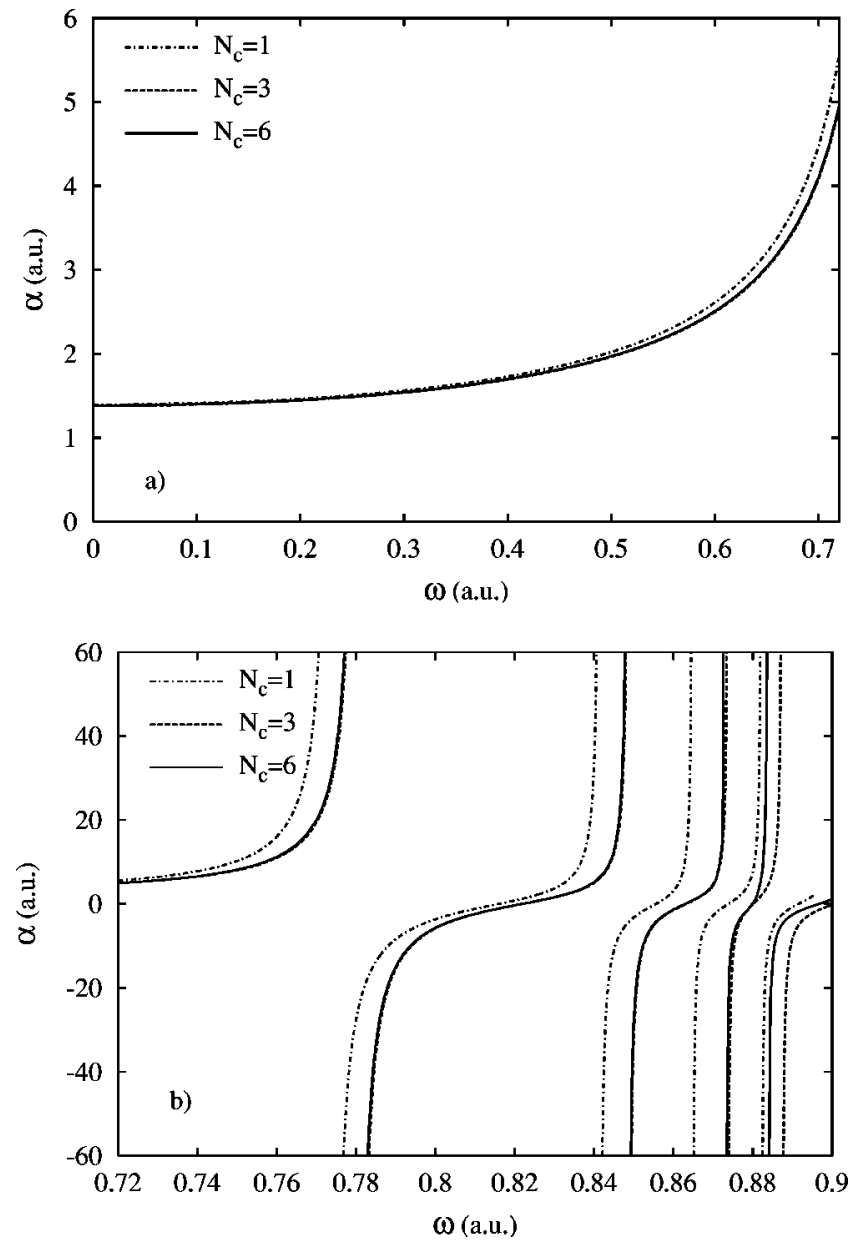

FIG. 3. Convergence of the dynamic polarizability of helium for photon frequencies below the first ionization threshold. (a) Below the first excitation frequency. (b) Resonance region.

by the values to the immediate left of the vertical lines. The numbers inside the boxes are the results after complete groups of channels in both ground and intermediate states are included. Thus, this analysis relates the convergence pattern of the polarizability with the behavior of the ${ }^{1} S^{e}$ and ${ }^{1} P^{o}$ energy convergence, shown in Tables I and II. The convergence with $N_{c}$ should be examined for entire groups of channels and the convergence with $N_{c}^{\prime}$ is monotonic and consequently one does not need to group channels, although one should expect jumps at the beginning of a new group. To further investigate the convergence and reliability of our results, an extrapolation procedure can be used. If one takes into account solely the results inside boxes from Table IV, each row and column can be fitted to a function of the type

$$
\alpha(x)=a+\frac{b}{x^{c}},
$$

whence one can extrapolate to an infinite number of groups in both directions. This extrapolation procedure may be done in two ways: starting by rows or by columns. Within six digits of accuracy, the resulting values are $\alpha_{\text {ext }}=1.38318$ and $\alpha_{\text {ext }}=1.38310$, respectively. Comparing these two ex-
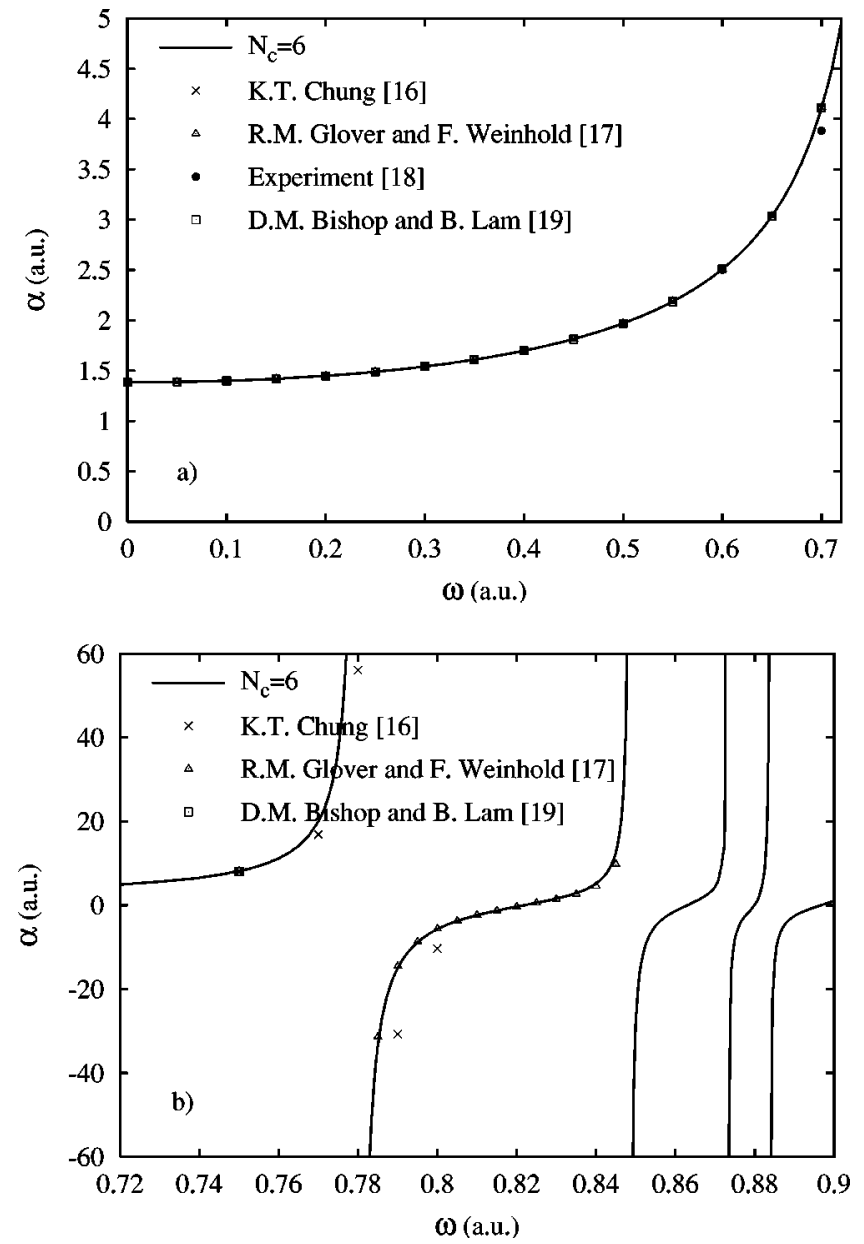

FIG. 4. Comparison of our best result for the dynamic polarizability of helium with other theoretical calculations and with experimental values. (a) Below the first excitation frequency. (b) Resonance region.

trapolated values with our result of $\alpha=1.383174\left(N_{c}=12\right.$ and $N_{c}^{\prime}=15$ ), we can assure a convergence of at least five digits. It is remarkable how our method is capable of providing results for polarizabilities that possess almost the same level of accuracy as for the ground-state energy (cf. Table I, $N_{c}=12$ ), which is usually not true.

In Table V, a comparison of our coupled channel values with the results of calculations of other authors and with experimental values is given. Our result using one optimized $\beta_{i}^{\mu}$ without logarithmic terms $\left(N_{c}=12\right.$ and $\left.N_{c}^{\prime}=15\right)$ and five nonoptimized $\beta_{i}^{\mu}$ including logarithmic terms $\left(N_{c}=N_{c}^{\prime}\right.$ $=12$ ) are presented. We also show the result of two optimized $\beta_{i}^{\mu}$ plus inclusion of logarithmic terms $\left(N_{c}=12\right.$ and $N_{c}^{\prime}=15$ ). As noted before, the improvement in the latter is beyond the sixth decimal digit. Not all of the results of others lie between the rigorous bounds from Glover and Weinhold [17]. Our results fall between these limits. Indeed, our precise results are comparable to the most accurate ones from the literature, such as that of Ref. [27]. Among the various theoretical calculations, the works by Bishop and co-workers [19,22], Bhatia and Drachman [23], Jamieson et al. [24], and Pachucki and Sapirstein [27] appear to be the most accurate 
TABLE VII. Comparison of the one-photon transition resonances extrapolated from Fig. 4(b) with results in the literature.

\begin{tabular}{ccc}
\hline \hline This work $^{\mathrm{a}}$ & Variational $^{\mathrm{b}}$ & Hyperspherical $^{\mathrm{c}}$ \\
\hline 0.780100 & 0.779881 & 0.779920 \\
0.848527 & 0.848578 & 0.848618 \\
0.872964 & 0.872655 & 0.872677 \\
0.883820 & 0.883818 & 0.883830 \\
\hline \hline
\end{tabular}

${ }^{\mathrm{a}} N_{c}=N_{c}^{\prime}=6$.

${ }^{\mathrm{b}}$ Reference [41].

${ }^{\mathrm{c}}$ Reference [42].

ones. Those are all based upon techniques that use variational wave functions for both the ground state and the intermediate states that involve large basis sets, ranging from 100 basis functions [22] to 900 [27]. Their wave functions do not include the logarithmic contributions, which can explain the large number of basis functions needed to achieve good convergence. Three of them $[22,23,27]$ used extended (or quadruple) numerical precision to avoid round-off error propagation. Nevertheless, our calculation using basis functions that include logarithmic terms needs fewer basis functions: namely, 60 terms in the expansions given by Eqs. (13) and (14), whereas in the calculation using only one optimized $\beta_{i}^{\mu}$ parameter, 16 terms $\left(M_{i}=16\right)$ have been used in these expansions. We reiterate that we have not pushed our variationally stable approach using a coupled adiabatic hyperspherical basis to obtain the seven or more digits of accuracy necessary to achieve benchmark predictions for the static polarizabilities. To do that we would have to couple more than the 12-15 channels of each symmetry that we currently include and, in particular, we would have to include one or more of the next complete groups of channels of each symmetry. What we have shown is that for a given number of channels the inclusion of logarithmic terms allows one to obtain comparable accuracy to calculations that optimize the $\beta$ parameters but do not use logarithmic terms. A key additional point is that the five digits of accuracy we have achieved at the level of approximation permitted by our computational constraints is sufficient to obtain competitive predictions for the dynamical polarizabilties, which we discuss next.

\section{B. Dynamic polarizability}

Our results for the static polarizability have determined the set of optimized parameters which provide results comparable to the use of logarithm terms in the initial- and intermediate-state expansions. We use this information to select the parameters for our calculations of the frequencydependent (or dynamic) polarizability of He. For the dynamic case, the determined optimal values of $\beta_{i}^{\mu}$ for each coupled channel in the static limit have been used. We have used an equal number of coupled channels for both ground and intermediate states, that is, $N_{c}=N_{c}^{\prime}=6$ since this number of coupled channels constitutes a closed group of channels for both ground and intermediate states, according to Table IV. In Table VI, our results for the dynamic polarizability of helium are compared with previous calculations in the literature [16-19] and also with experimental values (as listed in Ref. [18]). Most calculations are unable to achieve results over the photon energy range $0.75<\omega<0.9$ (in a.u.) owing to the approach of the resonance region. Our calculation for the dynamic polarizability is presented in Figs. 3 and 4. In Fig. 3 one sees the rate of convergence as the number of coupled channels (with $N_{c}=N_{c}^{\prime}$ ) increases. Figure 4 shows a comparison of our best result $\left(N_{c}=N_{c}^{\prime}=6\right)$ with the theoretical results of Chung [16], Glover and Weinhold [17], and Bishop and Lam [19] as well as with experimental values from Ref. [18]. For photon frequencies $\omega<0.7$ a.u. [Fig. $4(\mathrm{a})]$ our results compare very well with the results of other authors. However, in the resonance region [Fig. 4(b)] our calculation compares best with the results from Glover and Weinhold [17].

The resonances (sharp antisymmetric peaks) in the dynamic polarizability correspond to one-photon transitions to intermediate ${ }^{1} P^{o}$ excited states of He. When $\omega$ approaches this region ( $\omega \geqslant 0.7$ a.u.), conventional methods start to fail due to the approximation of the poles present in Eq. (1), making the calculation increasingly difficult. As our formalism transforms Eq. (1) into Eq. (2), in which the poles have been removed, there is no difficulty in the calculation in the region $\omega>0.7$ a.u. From the calculation of the dynamic polarizability in the resonance region, one can extract the frequencies for the one-photon transitions by simply inspecting the calculated data and, in the vicinity of an abrupt change of sign, extrapolate the values of the resonant frequencies. The first few one-photon transition energies obtained are listed in Table VII. In this table, a comparison with results of a variational calculation [41] is given. Also listed are the hyperspherical results from Ref. [42]. Unlike the usual methods for obtaining polarizabilities, one notes that the present variationally stable approach provides very accurate values for the one-photon transition energies.

\section{CONCLUSIONS}

In summary, we have presented a calculation for the static dipole polarizability of the helium ground state that includes logarithmic terms in both ground and intermediate states within a variationally stable, coupled-channel adiabatic hyperspherical approach. Within this basis set, the inclusion of logarithmic terms appears to be necessary to obtain convergence to the correct value of the static polarizability when nonoptimized parameters in the intermediate basis functions are used. We have shown that for any fixed number of coupled channels this approach is capable of obtaining a value for the static polarizability that is comparable to the results obtained with optimized parameters. The two methods appear comparable in their use of computer resources. Use of logarithmic terms requires more basis functions, but one does the calculation only once; use of optimized parameters - without logarithmic terms-implies fewer basis functions, but the calculations must be repeated as often as necessary to obtain the optimum values of the parameters.

Results for the dynamic polarizability have also been presented whose accuracy is comparable to that of the best results of other authors, especially in the photon frequency 
range corresponding to the one-photon transitions. In fact, one of the advantages of the present approach is the ability to furnish reliable and converged results even in the resonance region due to the nonexistence of poles in the transition matrix element. These accurate predictions for the dynamical polarizability imply that our approach is capable of predicting highly accurate values for multiphoton cross sections of helium (as well as other two-electron systems). Calculations for the helium two-photon ionization cross section are in progress.

\section{ACKNOWLEDGMENTS}

We thank G.W.F. Drake for valuable discussions and for a critical reading of an early draft of this paper. This work was supported in part by the U.S. Department of Energy, Office of Science, Division of Chemical Sciences, Geosciences, and Biosciences, under Grant No. DE-FG03-96ER14646. M.M. received financial support from Fundação de Amparo à Pesquisa do Estado de São Paulo (FAPESP)-Brazil, under Grant No. 99/11363-8.
[1] J.H. Bartlett, Phys. Rev. 51, 661 (1937).

[2] V.A. Fock, Izv. Akad. Nauk SSSR, Ser. Fiz. 18, 161 (1954) [K. Nor. Vidensk. Selsk. Forh. 31, 138 (1958); 31, 145 (1958)].

[3] A.M. Ermolaev and G.B. Sochilin, Dokl. Akad. Nauk SSSR, 155, 1050 (1964) [Sov. Phys. Dokl. 9, 292 (1964)].

[4] K. Frankowski and C.L. Pekeris, Phys. Rev. 146, 46 (1966).

[5] D.E. Freund, B.D. Huxtable, and J.D. Morgan III, Phys. Rev. A 29, R980 (1984).

[6] J.D. Baker, D.E. Freund, R.N. Hill, and J.D. Morgan III, Phys. Rev. A 41, 1247 (1990).

[7] H. Kleindienst and R. Emrich, Int. J. Quantum Chem. 37, 257 (1990).

[8] A.J. Thakkar and T. Koga, Phys. Rev. A 50, 854 (1994).

[9] G.W.F. Drake and Z.-C. Yan, Chem. Phys. Lett. 229, 486 (1994).

[10] S.P. Goldman, Phys. Rev. A 57, R677 (1998).

[11] G.W.F. Drake, Phys. Scr. T83, 83 (1999).

[12] G.W.F. Drake, M.M. Cassar, and R.A. Nistor, Phys. Rev. A 65, 054501 (2002).

[13] V.I. Korobov, Phys. Rev. A 61, 064503 (2000).

[14] V.I. Korobov, Phys. Rev. A 66, 024501 (2002).

[15] Yu.V. Popov and L.U. Ancarani, Phys. Rev. A 62, 042702 (2000).

[16] K.T. Chung, Phys. Rev. 166, 1 (1968).

[17] R.M. Glover and F. Weinhold, J. Chem. Phys. 65, 4913 (1976).

[18] E.-A. Reinsch, J. Chem. Phys. 83, 5784 (1985), Table I.

[19] D.M. Bishop and B. Lam, Phys. Rev. A 37, 464 (1988).

[20] B. Gao, C. Pan, C.R. Liu, and A.F. Starace, J. Opt. Soc. Am. B 7, 622 (1990).

[21] H.P. Saha and C.D. Caldwell, Phys. Rev. A 44, 5642 (1991).

[22] D.M. Bishop and J. Pipin, Int. J. Quantum Chem. 45, 349 (1993).

[23] A.K. Bhatia and R.J. Drachman, J. Phys. B 27, 1299 (1994).

[24] M.J. Jamieson, G.W.F. Drake, and A. Dalgarno, Phys. Rev. A 51, 3358 (1995).

[25] Z.-C. Yan, J.F. Babb, A. Dalgarno, and G.W.F. Drake, Phys. Rev. A 54, 2824 (1996).
[26] S.J.A. van Gisbergen, F. Kootstra, P.R.T. Schipper, O.V. Gritsenko, J.G. Snijders, and E.J. Baerends, Phys. Rev. A 57, 2556 (1998).

[27] K. Pachucki and J. Sapirstein, Phys. Rev. A 63, 012504 (2000).

[28] K.D. Bonin and M.A. Kadar-Kallen, Int. J. Mod. Phys. B 8, 3313 (1994).

[29] B. Gao and A.F. Starace, Phys. Rev. Lett. 61, 404 (1988).

[30] B. Gao and A.F. Starace, Phys. Rev. A 39, 4550 (1989).

[31] C.R. Liu, B. Gao, and A.F. Starace, Phys. Rev. A 46, 5985 (1992).

[32] M. Masili and A.F. Starace, Phys. Rev. A 62, 033403 (2000).

[33] U. Fano, Rep. Prog. Phys. 46, 97 (1983).

[34] J.M. Feagin, J.H. Macek, and A.F. Starace, Phys. Rev. A 32, 3219 (1985).

[35] J.E. Hornos, S.W. MacDowell, and C.D. Caldwell, Phys. Rev. A 33, 2212 (1986); 34, 2535 (1986).

[36] M. Masili, J.E. Hornos, and J.J. De Groote, Phys. Rev. A 52, 3362 (1995).

[37] C.D. Lin, Phys. Rep. 257, 1 (1995), and references therein.

[38] J.H. Macek, J. Phys. B 1, 831 (1968).

[39] A.F. Starace and G.L. Webster, Phys. Rev. A 19, 1629 (1979).

[40] H.T. Coelho and J.E. Hornos, Phys. Rev. A 43, 6379 (1991).

[41] A. Kono and S. Hattori, Phys. Rev. A 34, 1727 (1986).

[42] M. Masili, J.J. De Groote, and J.E. Hornos, J. Phys. B 33, 2641 (2000).

[43] B. Schiff, H. Lifson, C.L. Pekeris, and P. Rabinowitz, Phys. Rev. 160, A1104 (1965).

[44] C.R. Mansfield and E.R. Peck, J. Opt. Soc. Am. 59, 199 (1969).

[45] P.W. Langhoff and M. Karplus, J. Opt. Soc. Am. 59, 863 (1969).

[46] D. Gugan and G.W. Michel, Mol. Phys. 39, 783 (1980).

[47] D. Gugan, Metrologia 28, 405 (1991).

[48] K. Grohmann and H. Luther, Temperature-Its Measurement and Control in Science and Industry (AIP, New York, 1992), Vol. 6, p. 21. 